\title{
SWINBURNE ON THE SIMPLICITY OF THEISM
}

\author{
BRUCE LANGTRY \\ University of Melbourne
}

\begin{abstract}
This paper argues that (1) Richard Swinburne's general account of the simplicity of empirical hypotheses fails because it involves a deeply problematic notion of postulating a property, while there is a wide range of hypotheses where the assessment of simplicity rests entirely on the number and kinds of postulated properties (2) Swinburne's main argument in The Christian God for the simplicity of theism, the one based on considerations about pure limitless intentional power, is significantly weaker than he seems to believe. The paper does not draw a conclusion about whether theism is simple.
\end{abstract}

Section 1 of the paper introduces Swinburne's notion of simplicity as a multi-dimensional feature of a hypothesis, relevant to its intrinsic probability. Section 2 criticizes Swinburne's general theory of simplicity of hypotheses. Section 3 points out that even if theism is very simple, and God's necessary properties are very simple ones, it does not follow that God is ontologically simple. Section 4 identifies weaknesses in Swinburne's main argument in The Christian God for the simplicity of theism.

\section{H-SIMPLICITY AS A MULTI-DIMESNSIONAL FEATURE OF HYPOTHESES}

Swinburne's claim that theism is a very simple hypothesis plays an important role in his case for saying that the existence of God is more probable than not. ${ }^{1}$ For example, let e be the fine-tuning evidence, and let

\footnotetext{
${ }^{1}$ Swinburne's cumulative case is most fully set forth in The Existence of God, second edition, Oxford: Clarendon Press, 2004. The main conclusion is stated on p. 342. Since Swinburne himself holds (and has elsewhere argued for) the Christian doctrine of the
} 
$\mathrm{k}$ be all our admissible background evidence. Then according to Bayes' Theorem, the higher the intrinsic probability of theism is, the higher will be its probability relative to $\mathrm{k}$, and the higher its probability relative to $\mathrm{k}$ is, the higher will be its probability relative to e\&k. ${ }^{2}$ Swinburne identifies the two factors determining the intrinsic probability of a hypothesis as its simplicity and its scope, and he declares that simplicity is much more important than scope. ${ }^{3}$ Thus although there exist several different conceptions of simplicity (and degree of simplicity) of a hypothesis, what Swinburne has in mind is a logically necessary feature ${ }^{4}$, possessed independently of any auxiliary assumptions required to generate further hypotheses and predictions, and such that (jointly with scope) it determines the intrinsic probability of a hypothesis. ${ }^{5}$ I call this feature 'h-simplicity'.

Swinburne holds that h-simplicity is a multi-dimensional feature of theories:

[Quotation 1] The simplicity of a theory, in my view, is a matter of it postulating few (logically independent) entities, few properties of entities, few kinds of entities, few kinds of properties, properties more readily

Trinity, he explains on p. 344 that 'in Christian terms' his arguments in The Existence of God are arguments for the existence of God the Father.

2 In contexts like this, by 'probability' Swinburne means logical probability. The intrinsic probability of a proposition is its probability relative to a tautology.

${ }^{3}$ The Existence of God, pp. 53, 56; 'God as the Simplest Explanation of the Universe', European Journal for Philosophy of Religion 2 (2010), p. 5.

${ }^{4}$ In this paper 'logically necessary' and 'logically possible' are used broadly, as equivalent to 'metaphysically necessary' and 'metaphysically possible' respectively.

${ }^{5}$ Some people believe that the mathematical discipline information theory provides a good criterion of h-simplicity. For example, David L. Dowe, Steve Gardner and Graham Oppy, 'Bayes not Bust! Why Simplicity is no Problem for Bayesians', British Journal for Philosophy of Science 58 (2007), pp. 709-754. The first paragraph of the paper makes it clear that the authors intend to discuss the concept of simplicity which is most directly relevant to arguments from empirical evidence. As far as I know, however, informationtheoretic proposals have so far been developed only with respect to the curve-fitting problem, whereas discussing h-simplicity more generally, and theism as an empirical hypothesis specifically, requires far more broadly applicable criteria. Elliott Sober holds that the simplest hypothesis is 'the one with respect to which you need to obtain less additional information in order to answer your questions' (Simplicity, Oxford: Clarendon Press, 1975). Swinburne replies that this concept of simplicity is irrelevant to probable truth (Epistemic Justification, p. 86n). 
observable $^{6}$, few separate laws with few terms relating few variables, the simplest formulation of each law being mathematically simple. ${ }^{7}$

[Quotation 2] One formulation of a law is mathematically simpler than another in so far as the latter uses terms defined by terms used in the former but not vice versa ... ${ }^{8}$

[Quotation 3] Hypotheses attributing infinite values of properties to objects are simpler than ones attributing large finite values. ${ }^{9}$

[Quotation 4] The same hypothesis, in the sense of one postulating the same entities, properties and relations between them, may be expressed in logically equivalent formulations ... One hypothesis is simpler than another if (and only if) the simplest formulation of the former is simpler than the simplest formulation of the latter. ${ }^{10}$

${ }^{6}$ Although a proposition's degree of h-simplicity must be a necessary matter, since it is to be a guide to its intrinsic probability, in the most common contexts in which 'observable' is used whether one property counts as more readily observable than another is typically a contingent affair, depending on the perceptual capacities with which humans are naturally endowed, and on the availability of devices such as telescopes and processes such as magnetic resonance imaging. In Epistemic Justification, p. 89, Swinburne recognizes this, and argues that nevertheless 'there remains a logical dependence of the observability of some properties on the observability of others'. It seems to me that if Swinburne is to sustain this claim, without relativizing observability (and so h-simplicity) to natural endowments of different species of intelligent beings, he can do so only by supposing that various laws of nature are logically necessary truths - e.g., laws about wavelengths of radiation which could be used in direct perception.

${ }^{7}$ The Existence of God, p. 53. Swinburne adopts the same account on p. 273 of his 'A simple theism for a mixed world: response to Bradley', Religious Studies 43 (2007), pp. 271-277, and on pp. 25, 29 of Is There a God?, revised edition, Oxford University Press, 2010. Notice that although in Quotation 1 Swinburne uses the word 'theory', which suggests a changing cluster of propositions, some more central and resistant to revision than others, the 'theism' whose h-simplicity Swinburne discusses is the single proposition There is a God, understood as he understands it. (The Existence of God, pp. 7, 344, The Christian God, p. 126, and 'A Simple theism for a mixed world: response to Bradley', pp. 73-274). One might naturally take a theistic theory of some subject matter to consist of the conjunction of There is a God with, for example, various controversial value propositions, and propositions specifying whether God causally determines all events our universe, but Swinburne does not for the most part discuss the degrees of simplicity of such wholes.

8 The Existence of God, p. 54

9 The Existence of God, p. 55.

${ }^{10}$ Epistemic Justification, p. 87. 
[Quotation 5] It is always simpler to postulate an absence than a presence. ${ }^{11}$ [Quotation 6] A property defined by similarity to paradigm examples of its application, such as 'green' or 'mass' or 'bright' counts as one property; properties defined as conjunctions or disjunctions of such properties (or as having more complicated probabilistic relations to such properties) count as two or more properties. ${ }^{12}$

[Quotation 7] Where there is a possibility of higher-level hypotheses [which might explain medium level hypotheses postulated to explain some collection of evidence] the extent to which medium-level hypotheses might fit with other medium-level hypotheses into a higherlevel hypothesis is relevant to determining their simplicity. ${ }^{13}$

How are we to interpret Quotation 4? On the one hand, there is a reason to think that the phrase 'the simplest formulation' cannot mean the h-simplest formulation. Since distinct formulations of a hypothesis are distinct propositions, it follows that if it is a priori that two formulations are logically equivalent to each other while differing with respect to h-simplicity, then either there is some precisely offsetting difference in scope between them, or else they differ in intrinsic logical probability. It is hard to believe that there will always, or even typically, be a precisely offsetting difference in scope. But if these two formulations differ in intrinsic probability, then we have a major departure from the probability calculus - a departure which Swinburne would be reluctant to make.

On the other hand, what might Swinburne mean by 'the simplest formulation' other than the h-simplest one? Apart from those cases in which the two formulations differ merely in degree of mathematical simplicity, he gives no indication that he has in mind any other account of simplicity of formulations. Accordingly, here is a plausible interpretation of Quotation 4:

(Z) Consider any set $\mathrm{S}$ of contingent propositions such that for any two members $\mathrm{p}$ and $\mathrm{q}$, it is an a priori truth that $\mathrm{p}$ is logically equivalent to $\mathrm{q}$. The members of $\mathrm{S}$ are to be regarded as formulations of one hypothesis. We should apply the criteria given in Quotations $1-7$

\footnotetext{
11 'God as the Simplest Explanation of the Universe', p. 19.

12 'God as the Simplest Explanation of the Universe', p. 7.

13 'Gwiazda on the Bayesian argument for God', Philosophia 39 (2011), pp. 393-396.
} 
to each member of $S$ to judge which is the h-simplest, and regard the degree of h-simplicity of this one as measuring (i.e., as equal to) the degree of h-simplicity of the hypothesis.

Given this interpretation, should we identify the hypothesis with its h-simplest formulation? If not, then with what should we identify it? Perhaps Swinburne should regard a hypothesis as not a proposition but a set $S$ of propositions all and only of which are logically equivalent to each other; the members of $S$ are said to be formulations of $S$, and any sentence which expresses a member of $S$ is said to express $S$. Swinburne gives no sign that this is in fact how he should be understood. But consider the alternative, that a hypothesis $h$ is itself a proposition. In that case, the formulations of $\mathrm{h}$ would be all and only those propositions such that it is a priori that they are logically equivalent to $h$. Since the relation between $\mathrm{h}$ and its formulations seems symmetrical, surely nothing would stop us from saying that $h$ is a formulation of each of these other propositions. So according to this alternative account, the word 'formulation' is idle, and can be dropped without loss. One could replace $\mathrm{Z}$ by $\mathrm{Z}^{\star}$ :

$\left(Z^{\star}\right)$ Consider any set $S$ of contingent propositions such that for any two members $\mathrm{p}$ and $\mathrm{q}$, it is an a priori truth that $\mathrm{p}$ is logically equivalent to q. We should proceed in two stages: first, apply the conditions stated in Quotations 1 - 7 to each member of $S$ to judge which one fulfils them to the greatest extent; second, treat the extent to which this member fulfils the conditions as measuring the degree of h-simplicity of each member of S.

Since $Z^{\star}$ departs far from the way Swinburne expresses himself in Quotation 4 and elsewhere, it amounts to a friendly proposal for replacing Swinburne's actual view with a rational reconstruction of it, rather than an interpretation of it. Moreover, adopting $\mathrm{Z}^{\star}$ would inconveniently complicate the composition of subsequent discussion of what Swinburne says. So let us work with Z.

Quotation 6 offers us little help with respect to the general theory of h-simplicity, because of difficulties in counting properties. Being a bachelor can perhaps be understood as not a single property but as the conjunction of two putative properties, being unmarried and being a man. Yet it can equally well be understood as the conjunction of four putative properties, being unmarried, being an adult, being male, and 
being a human. And since individual animals of a wide variety of species are classified as male, it would require a lot of a priori reflection (and perhaps empirical investigation) to determine whether being male is a single property or a conjunction of properties, and if a conjunction of properties how many conjuncts there are. So if we want to compare the two rival hypotheses This is a star and This is a planet, even roughly, with respect to the number of properties postulated, realizing that 'star' and 'planet' are defined by astronomers today differently to the way they were understood 1000 years ago, then how are we to go about counting?

Quotation 7 is puzzling, because most of the examples of scientific hypotheses which Swinburne gives in his discussions of h-simplicity in Epistemic Justification and The Existence of God are medium-level ones where obviously there will be higher-level hypotheses, true ones and false ones, which purport to explain them - e.g., One puma escaped from captivity, Quarks have spin, Kepler's three laws of planetary motion, and All ravens are black. ${ }^{14}$ Each logically possible candidate explanans must somehow count, since h-simplicity, being a logically necessary feature of a hypothesis, cannot depend on what is contingently the case. Swinburne does not explain how the infinitely many candidate explanans for (say) Kepler's three laws of planetary motion, candidates which no doubt differ widely in h-simplicity, contribute to fixing the degree of h-simplicity of these three laws.

\section{POSTULATING PROPERTIES}

This section begins with some remarks about how Swinburne uses the word 'property'. At some places he says that he is doing so in such a way that every predicate designates a property (except for predicates of the form 'is identical with individual $a$ '), and in such a way that any two nonsynonymous predicates designate distinct properties. ${ }^{15}$ Thus he would

\footnotetext{
${ }^{14}$ Epistemic Justification, pp. 87, 89f, 91.

${ }^{15}$ The Christian God, pp. 10, 34; cf. The Existence of God, p. 41. Swinburne's liberal use of 'property' may encounter difficulties with predicates such as 'heterological. (A predicate is heterological if and only if it is not true of itself - e.g., 'dyadic' is heterological.) Furthermore, while the verb 'loves' is a many-place predicate, it is not clear that Helen loves Zeus, which does not assert or entail the existence of Zeus, should count as affirming
} 
say that the properties being sulphuric acid and being $\mathrm{H}_{2} \mathrm{SO}_{4}$ are distinct, even though they make the same contribution to the way things behave.

As I have already indicated, Swinburne modifies his view that nearly every predicate designates a property when he says that some predicates designate conjunctions or disjunctions of properties instead. More significantly, Swinburne does not always use 'property' in such a way that any two non-synonymous predicates designate distinct properties. For he argues that on his preferred understandings of the predicates used in his explanation of the meaning of 'There is a God', they fit together to designate the one simple property, having pure, limitless, intentional power. ${ }^{16}$ The conjunction of 'is omnipotent,' 'is omniscient,' etc, is not synonymous with 'has pure, limitless, intentional power'. After all, when Swinburne argues that the property Pure, limitless, intentional power entails perfect goodness, his argument employs the premise that agents perform no actions which they believe to be overall bad, and do what they believe to be a best action (or best kind of action) if there is one, unless they are subject to non-rational influences. ${ }^{17}$ This premise, even if it is necessarily true, is not analytic. (From now on, the predicate 'has pure, limitless, intentional power will be abbreviated as 'has plip', while the corresponding singular term purporting to refer to a property will be abbreviated as 'Plip.')

One might reasonably suppose, given a liberal attitude to the existence of properties, that a hypothesis postulates a property designated by the predicate 'is F' if and only if it asserts or entails that there is something of which 'is F' is true. Thus if a scientific theory endorses the ideal gas law, but does not entail that there are any ideal gases, then it does not postulate the property being an ideal gas.

Swinburne, however, does not and cannot say this. Consider the hypotheses There are no mature vertebrates with two hearts and If there are black holes then they all emit Hawking radiation. Plainly, neither

that Helen stands in the relation loves to Zeus, and thereby postulating this relation instead of (or as well as) the monadic property loving Zeus.

${ }^{16}$ The Christian God, p. 126. On p. 151 he speaks of the 'reduction' of being bodiless, omnipresent, perfectly good, etc, to being necessarily perfectly free, omniscient and omnipotent.

${ }^{17}$ The Christian God, p. 151. 
of them entails the existence of any entities or the truth of any laws. ${ }^{18}$ So according Quotations 1 - 6, the considerations relevant to their degrees of h-simplicity are reduced to how many properties each of them respectively postulates, how many kinds these properties fall under, and how readily observable the properties are, and whether the properties are said to involve infinite magnitudes. But neither hypothesis entails that any property is instantiated. Nor does Quotation 7, or Swinburne's accompanying remarks, provide a way in which we can assess the degree of simplicity of either hypothesis. ${ }^{19}$

Thus Swinburne's general theory of h-simplicity must not incorporate the assumption that postulating a property is a matter of asserting or entailing that the property is instantiated. This truth, however, turns out to generate serious difficulties for the theory. Consider the following candidate principle:

(C1) If $\mathrm{p}$ and $\mathrm{q}$ are contingent, and $\mathrm{p}$ entails $\mathrm{q}$, and $\mathrm{q}$ postulates the property Fness then p postulates the property Fness.

There are two strong reasons why, given the points made in the preceding paragraph, Swinburne should reject $\mathrm{C} 1 .^{20}$ Firstly, $\mathrm{C} 1$ is incompatible with the conjunction of two principles which he has strong reasons for accepting, namely:

(C2) In general, if the hypothesis Eitherp or there are Fs is contingently true or false then it postulates the property Fness. ${ }^{21}$

${ }^{18}$ Although the expressions 'black hole' and 'Hawking radiation' are theoretically laden, it does not follow that the second hypothesis entails, e.g., the General Theory of Relativity. Suppose that you assert 'If phlogiston had existed then Lavoisier would have been wrong.' The word 'phlogiston' is theory-laden, in the sense that someone who did not know (for example) that phlogiston was believed to be liberated from burning objects would have only a very limited grasp of the meaning of the word, but the conditional proposition you assert does not entail any propositions which, if they were true, would be laws.

${ }^{19}$ Given Quotation 7, each logically possible candidate explanans must somehow count, since h-simplicity, being a logically necessary feature of a hypothesis, cannot depend on what is contingently the case. But how do they count?

${ }^{20}$ In personal communications, Swinburne has said that he rejects C1.

${ }^{21} \mathrm{C} 2$ should be understood as tacitly excluding certain substituens for ' $\mathrm{F}$ ', such as 'predicate which is not true of itself'. The word 'hypothesis' in C2 should be understood as excluding occurrences of Either $p$ or there are Fs in such propositions as Alice believes that either $p$ or there are Fs. 
(C3) It is not the case that any arbitrarily selected contingent hypothesis postulates any arbitrarily selected property. Proof:

(a) Either snow is white or there are protons postulates being a proton [from C2]

(b) Snow is white is contingent and entails Either snow is white or there are protons [necessarily true premise]

(c) Snow is white postulates being a proton [from a, b, C1]

but c) is obviously ruled out by $\mathrm{C} 3$.

$\mathrm{C} 3$ is secure for obvious reasons. C2 is motivated by the existence of pairs of disjunctive empirical hypotheses (not entailing the existence of any entities, postulating any laws, or employing any quantitative expressions, and not differing with respect to distance of postulated properties from observation) whose degree of h-simplicity one might want to compare for example, (i) Either there are no black holes, or else there are black holes and each of them emits Hawking radiation and (ii) Either there are no black holes, or else there are black holes and they include very short-lived ones which do not emit Hawking radiation.

Assuming that Swinburne is committed to saying that both (i) and (ii) postulate such properties as being a black hole and emitting Hawking radiation, is there nevertheless some way in which he can maintain that Either snow is white or there are protons does not postulate being a proton? It is hard to see how some difference in content could generate such a difference between (i) and (ii) on the one hand and Either snow is white or there are protons on the other hand. If there is indeed such a difference then it seems that it must rest not on differences in their content but instead on the reasons why each proposition is asserted, denied, accepted, rejected, regarded as worthy of further consideration, and so on. But if the various features listed in Quotations 1 - 7 are to contribute to fixing degree of h-simplicity and thereby intrinsic probability, then they must be matters merely of content.

The second reason why Swinburne should not adopt $\mathrm{C} 1$ relies on the assumption, already discussed, that at least some hypotheses postulate a property designated by a predicate occurring in them even though they do not entail that the property is instantiated. Given this assumption, it is plausible to suppose that There is a being who is omnipotent, 
omniscient, etc ${ }^{22}$ entails God has the power to prevent its being the case that there are tennis players, unicorns, etc. Therefore if $\mathrm{C} 1$ were true then theism would postulate every property postulated by the latter proposition, including being a tennis player and being a unicorn. In that case, Swinburne's arguments for the h-simplicity of theism, employing the criteria supplied by Quotations 1 - 7, would be undermined.

So Swinburne should reject C1. Doing so, however, would cripple his theory of h-simplicity. Assume for the sake of discussion that it is straightforwardly true that This tablet contains aspirin postulates the property containing aspirin, and This tablet contains an acid postulates the property containing an acid, and This tablet contains a drug postulates the property being a drug. Yet in the absence of $\mathrm{C} 1$ he seems to have no grounds on which to say that This tablet contains aspirin postulates the properties containing an acid and containing a drug (along with other properties such as being an analgesic and occupying space). ${ }^{23}$ Thus the resulting Swinburnean account would place great emphasis on the difference between what The tablet contains aspirin asserts and what it entails. But why should we suppose that this difference makes a difference to h-simplicity and thereby to intrinsic probability? That is, why, with respect to The tablet contains aspirin, is the number of properties postulated (and the kinds the properties fall under), thus narrowly construed, what counts towards the proposition's h-simplicity and intrinsic probability? ${ }^{24}$

Furthermore, if $\mathrm{C} 1$ is rejected, then how is Swinburne to use Quotations 1 - 7 to compare, with respect to h-simplicity, The object is a star with The object is a planet, and The sample contains human sweat with The sample contains a pesticide? It is easy enough to imagine contexts of inquiry with respect to which the second of these comparisons would be useful. For example, the two hypotheses might be rival explanations of the presence in the sample of some specific molecule which is often present in human sweat and in pesticides, but which rarely occurs

\footnotetext{
${ }^{22}$ I will use the sentence 'There is being who is omnipotent, omniscient, etc' as short for the sentence $\mathrm{T}$ in the Quotation 8 below.

${ }^{23}$ Dictionary definitions of 'aspirin' commonly specify that aspirin is an analgesic, and that the chemical name for aspirin is 'acetylsalicylic acid'.

${ }^{24}$ The example cannot be dismissed as a marginal one. Similar points would apply if we took as our example the hypothesis Rover is a dog.
} 
elsewhere; we can suppose that the presence of the molecule is equally probable relative to the conjunction of each hypothesis with background evidence; our assessment of which hypothesis was more probable relative to background evidence alone might depend largely on which hypothesis we regarded as the h-simpler.

Here is another way of putting the foregoing points. Suppose, to simplify a little, we accept that 'aspirin' is defined as 'acetylsalicylic acid'. How does this definition enable us to apply the insights provided in Quotations 1 - 7 to This sample contains aspirin and thereby estimate this hypothesis' degree of h-simplicity? We might try unpacking the definiens: when we say that aspirin is an acid, and is an acetylsalicylic substance, how complicated is what we are saying? This depends on how complicated the notion of an acid is, and the notion of an acetylsalicylic substance is. Yet surely the difference between truths guaranteed by chains of definitions and truths guaranteed by other forms of a priori reasoning cannot be fundamental to the assessment of h-simplicity. If how h-simple This sample contains aspirin is depends to a considerable extent on how many properties it postulates, then every property $\mathrm{F}$ such that it is a priori that This sample contains aspirin entails This sample contains something $F$ should be counted. Yet how can we continue to maintain this is we reject $\mathrm{C} 1$ ? The aspirin example cannot be dismissed as one of a few difficult cases in which we cannot estimate how h-simple the hypothesis is; the same point applies very widely.

I conclude that Swinburne's general account of h-simplicity is seriously defective. It might, however, be suggested that for the purposes of assessing the h-simplicity of theism Swinburne can employ a restricted account of h-simplicity. Let us say that a hypothesis is existential if and only if it asserts or entails the existence of at least one non-abstract entity, and let us say that one existential hypothesis is more economical than another with respect to natural kinds of entities, causal powers, etc, if and only if the former entails the instantiation of fewer natural kinds than the latter does, and the possession by entities of fewer causal powers than the latter does. The current suggestion is that Swinburne could say merely that for existential hypotheses what counts towards h-simplicity is economy with respect to entities, natural kinds of entities, fundamental causal powers and (in the case of rational agents) beliefs, desires and intentions, along with mathematical simplicity. 
Perhaps something along these lines can be worked out. Here, however, is an elementary objection with which any such restricted account would have to deal. Consider the hypothesis (iii) There are neutron stars, and either there are no black holes or else there are black holes and each of them emits Hawking radiation. It is an existential hypothesis, since it entails the existence of neutron stars and the instantiation of such natural kinds as Mass-possessing entity. But plainly (iii) is no less economical than There are neutron stars is, with respect to entities, natural kinds of entities, fundamental causal powers, beliefs, desires and intentions, and the two do not differ in mathematical simplicity. So according to the proposed restricted account, (iii) does not differ in degree of h-simplicity from There are neutron stars. This result, however, reveals that the proposed account is no mere restriction of Swinburne's actual theory to cover merely a sub-class of hypotheses. It is contrary to the spirit of Swinburne's theory.

\section{ONTOLOGICAL SIMPLICITY, AND ITS RELATION TO H-SIMPLICITY}

Consider inferences from h-simplicity to ontological simplicity. It is logically necessary that a specific hypothesis has the degree of h-simplicity it has, while in a great many cases at least, degree of ontological simplicity/ complexity is a contingent feature of a thing. Therefore the truth that There are Fs is h-simple will not typically entail that some specified F, let alone all Fs, are ontologically simple. After all, often Fness will be only one of many properties of each actual $\mathrm{F}$ thing. Although, no doubt, There are horses is h-simpler than There are insects with genetic code such-and-such (where 'such-and-such' is short for a detailed specification), every insect with the specified genetic code is simpler than any horse.

How about inferences in the opposite direction, from ontological simplicity to h-simplicity? If There are Fs is h-simpler than There are Gs, it is necessarily so. Therefore, in classical logic, if There are Fs is $\mathrm{h}$-simpler than There are Gs then Any $\mathrm{F}$ is ontologically simpler than any $G$ entails that it is h-simpler. Nevertheless, in general, if we are trying to discover whether There are Fs is h-simpler than There are Gs, does Any $F$ is ontologically simpler than any $G$ give us a reason to believe that it is? I am not aware of a good argument for supposing that the answer must 
be Yes, and there is a good direct reason for supposing that the answer is No: Assume that, as a matter of contingent fact, any insect is ontologically simpler than any horse. It follows by elementary logic that any insect with genetic code such-and-such is ontologically simpler than any horse. Obviously this entailed truth gives us very little reason to believe that There are insects with genetic code such-and-such is h-simpler than There are horses.

Swinburne frequently says that God is a being of a very simple kind; perhaps at least some of these statements should be read as declarations that God is ontologically very simple. ${ }^{25}$ There are surprisingly few other explicit statements in Swinburne's writings to this effect. For reasons given above, even if There is a God is very h-simple, and God's de re necessary properties are very simple ones, it does not follow that God is very ontologically simple.

\section{SWINBURNE'S ARGUMENT CONNECTING THE SIMPLICITY OF PLIP AND THE H-SIMPLICITY OF THEISM}

\section{In The Christian God, Swinburne says:}

[Quotation 8] The claim that there is a God is to be understood, provisionally, as the claim [T] that there exists necessarily and eternally a person essentially bodiless, omnipresent, creator and sustainer of any universe there may be, perfectly free, omnipotent, omniscient, perfectly good, and a source of moral obligation. ${ }^{26}$ On certain understandings of the foregoing predicates, they fit together so as together to designate one very simple property, of having (necessarily) pure, limitless intentional

${ }^{25}$ Such declarations seem to occur in The Existence of God, p. 147 (interpreting 'such' to refer to 'being' rather than 'explanation'), in The Christian God, p. 160, and in 'God as the Simplest Explanation of the Universe', p. 20. The proposition that God is a person of a very simple kind need not, of course, be read as entailing that God is ontologically very simple. After all, Swinburne's arguments surely establish at most that the conjunction of all God's essential properties constitutes a very simple kind.

${ }^{26}$ Care is required to interpret Swinburne's account in accordance with his sometimes surprising explanations, for example, 'By saying that God is essentially bodiless, I mean that, although he may sometimes have a body, he is not dependent on his body in any way.' The label ' $\mathrm{T}$ ' is mine. 
power. It follows that the claim that there is a God is a very simple claim and hence much more likely to be true. ${ }^{27}$

[Quotation 9] I conclude that the existence of a substance who has necessarily pure, intentional, limitless power entails and is entailed by the existence of a substance who has necessarily the divine properties [designated in T]. I understand by a divine individual one who has necessarily pure, intentional, limitless power. The claim that there is a God is therefore to be read as the claim that there is such an individual. ${ }^{28}$

Thus prompted, let us consider the following, which I will call the Plipbased argument:

(1) Plip is a very simple property.

(2) Hence There is an entity which has plip is very h-simple.

(3) It is an a priori truth that There is a God is logically equivalent to There is an entity which has plip..$^{29}$

(4) Hence There is a God is very h-simple. ${ }^{30}$

I will state and discuss three objections to the Plip-based argument. The first casts doubt on the inference from (2) and (3) to (4). Recall Quotation 4 , and my interpretation of it as asserting Z. Given Z, it follows from (3) that the propositions There is a God and There is an entity which has plip are two different formulations of one hypothesis. No doubt there are other formulations. If we were to accept (2) then we might conjecture that There is an entity which has plip is the h-simplest of all the formulations, and proceed to identify the degree of h-simplicity of the hypothesis with the degree of h-simplicity of There is an entity which

${ }^{27}$ The Christian God, pp. 125-126. These three sentences closely paraphrase Swinburne.

${ }^{28}$ The Christian God, p. 157.

${ }^{29}$ Swinburne asserts this premise in The Christian God, p. 157. In various passages near Quotation 8, Swinburne sounds as if he is claiming merely that (it is a priori that) Plip entails having omnipotence, omniscience, etc. But in general, the schema $A$ and $B$ are contingent, $A$ is $h$-simple, and it is a priori that $A$ entails $B$; therefore $B$ is $h$-simple is invalid. A case in which truth is not preserved is obtained by letting A be This object is spherical and letting B be Either this object is spherical or else our instruments have such-and-such a complicated technical fault.

${ }^{30}$ How simple is very simple? We should bear in mind the role of propositions like (4) in assessments of empirical arguments for the existence of God. (4) can be understood as the claim that There is a God is much h-simpler than any rival which has anywhere nearly as much, or more, explanatory power with respect to evidence statements such as The observed universe is fine tuned. 
has plip. But none of this suffices to exclude the conjecture that There is a God is very h-complex. Indeed, if on the basis of Quotation 8 we were to identify There is a God with T, and if (ignoring my criticisms of Swinburne's general theory of h-simplicity) we were to apply the criteria of h-simplicity given in Quotations 1 - 7 to T, and we were to accept that it is an a priori truth that $\mathrm{T}$ is logically equivalent to There is an entity which has plip, then we would have to say that There is a God is a less-than-maximally h-simple formulation of the hypothesis of which There is an entity which has plip is an h-simpler formulation. The same point would hold if we were to replace the criteria given in Quotations $1-7$ by some currently unformulated criterion restricted to existential propositions.

It might be protested that Quotation 8, from The Christian God, identifies There is a God with There is an entity which has plip, and that given this identification, (2) by itself suffices to entail (4).This point succeeds in defending the Plip-based argument against the first objection if and only if it is reasonable to identify There is a God with There is an entity which has plip. ${ }^{31}$ It is unlikely, however, that Swinburne himself currently feels committed to the identity. The expression 'pure limitless intentional power' does not occur either in The Existence of God, or in Is There $a$ God? ${ }^{32}$ or in 'God as the Simplest Explanation of the Universe'33. His explanations of There is a God which are more recent than those in A Christian God $^{34}$ are in terms very similar to T. Although Plip plays an important role in 'How the divine properties fit together' ${ }^{35}$, which deals with divine ontological simplicity, the paper does not say anything that suggests he still wants to maintain the identity. I can see no reason why we ourselves should do so. After all, we do not in general identify $\mathrm{p}$ with q given merely that it is a priori that $\mathrm{p}$ is logically equivalent to $\mathrm{q}$.

${ }^{31}$ Such an identification might be presented not as involving a claim of synonymy but instead as an explication, along the lines of the identification of the ordered pair $\langle\mathrm{x}, \mathrm{y}\rangle$ with the set $\{\{x\},\{x, y\}\}$, where alternative explications would also have been viable.

${ }^{32}$ Second Edition, Clarendon Press 2010.

${ }^{33}$ European Journal for Philosophy of Religion 2 (2010), 1-24

${ }^{34}$ E.g., in The Existence of God, p. 7.

35 'How the divine properties fit together: reply to Gwiazda', Religious Studies 45 (2009), pp. 495-498. 
Someone might respond to the first objection by abandoning (1)-(4), and replacing it by a closely related argument which, the responder hopes, will deliver much but not all of what the Plip-based argument was intended to achieve. Let us start with $\mathrm{T}$ and consider the various propositions including There is an entity which has plip - each of which is such that it is a priori that it is logically equivalent to T. In the light of $\mathrm{Z}$, each of these propositions should be regarded as a formulation of one hypothesis, to which we can give the name 'theism', and theism will be either just as simple as or even simpler than There is an entity which has plip. Hence if There is an entity which has plip is h-simple then theism is h-simple. It does not matter much what we say about the specific formulation There is a God. The revised Plip-based argument succeeds in avoiding the first objection, though it still encounters the other two objections stated below, and obviously it departs far from the letter of what Swinburne says.

Let us move on to the second objection to the Plip-based argument, which casts doubt on the inference from (1) to (2). It begins by pointing out that the following principle is not true:

(N) Necessarily, if There is an entity which is $F$ is the h-simplest formulation of a hypothesis then the degree of simplicity of the property designated by the predicate 'is $\mathrm{F}$ ' is proportional to the degree of h-simplicity of There is an entity which is $F$, and vice versa. ${ }^{36}$ Notice that Quotations 1 - 7 do not entail or in any other way commit Swinburne to $\mathrm{N}$ because none of them incorporate any considerations about simplicity of properties. Given that Swinburne should reject C1, then at least until he develops a theory of the simplicity of properties - he has not so far done so $^{37}$ - he should reject N. For it is intuitively

\footnotetext{
${ }^{36}$ Perhaps some principle like N can be dimly glimpsed behind Swinburne's apparent inference, in The Existence of God, p. 97, from The hypothesis that God is omnipotent, omniscient etc is very h-simple to God's intentions, beliefs and basic powers are of a very simple kind.

${ }^{37}$ Quotation 6 is relevant, but does not get us very far. After all, there seem to be pairs of properties (e.g., being a woman, being a proton: many two-year olds can reliably identify women) such that the one more accessible to observation is more complex than the other one; and if this is not so then it needs to be explained why not. Alongside Quotation 6, it is worth noticing a passage in An Introduction to Confirmation Theory, Methuen 1973, p. 148, where Swinburne says in effect that that he understands ' $\mathrm{P}_{1}$ is a simpler predicate than $\mathrm{P}_{2}$ ' to mean that that universal nomological propositions which
} 
plausible that being cancerous is a much more complex property than being perfectly spherical. But given that $\mathrm{C} 1$ should be rejected, each of the propositions There is an entity which is cancerous and There is an entity which is perfectly spherical postulates at most one entity, and at most one property, and since each of them is non-quantitative, and since being cancerous and being perfectly spherical do not differ much in ease of observability, then, in the absence of any reason for thinking that either of the propositions is a formulation of some hypothesis which has a simpler formulation, Swinburne's criteria of h-simplicity commit him to regarding the two propositions as equally h-simple, or as differing little in degree of h-simplicity.

Assuming that $\mathrm{N}$ is not true, why should we believe that (1) is a good reason for (2)? Any answer would have to appeal to some distinctive feature with respect to which Plip, i.e., the property designated by 'has plip', differs relevantly from other properties designated in other hypotheses. Unless Swinburne can identify such a feature, and show that it explains why the inference from (1) to (2) differs from otherwise similar inferences, we have no reason for accepting that (1) supports (2).

The third objection to the Plip-based argument concerns the inconclusive character of Swinburne's case for the truth of (1). Sometimes he seems to be arguing from the h-simplicity of the hypothesis that God is omnipotent to the simplicity of the property omnipotence, and so on for other divine properties. ${ }^{38}$ If he were relying on this line of thought alone, however, then the overall argument in which the Plip-based argument is nested would be circular.

He provides other support for the truth of (1) in The Christian God, pp. 151-152. Plip, he says, is to be understood in accordance with the premises of the argument below:

(5) Having plip involves all the agent's causing being intentional: everything which the agent, $\mathrm{x}$, brings about he means to bring about, and hence $\mathrm{x}$ acts only on reason.

use $\mathrm{P}_{1}$ are h-simpler, as judged by his criteria of h-simplicity, than universal nomological propositions which use $\mathrm{P}_{2}$. This remark is not helpful in the present context, where we are assessing an inference from (1) to (2), rather than from (2) to (1), and where the inference from (1) to (2) is intended to significantly strengthen his case for the h-simplicity of theism.

${ }^{38}$ The Existence of God, p. 97. 
(6) The power is limitless in that all events that occur (other than x's existing), can do so only because of x's currently either bringing them about or allowing some other substance to bring them about; and all events that do not occur do not occur for that same reason. X's exercise of power is not limited by any ignorance of what $\mathrm{x}$ can do with it, or by any substance from without causally influencing how $\mathrm{x}$ acts

(7) Therefore, intuitively the notion of plip is very simple. Inferring (7) from (5) and (6) is perhaps motivated by the thought, expressed in Quotation 3 and reflecting patterns in theory-choice by scientists and other empirical investigators, that, other things being equal, hypotheses which attribute limitlessness are h-simpler than those which attribute limits. But even if we add this thought as a premise alongside (5) and (6), the three premises will not jointly entail (7). Alternatively, someone might seize on the word 'intuitively' in (7) and claim that our intuitions favour the view that Plip, as characterized by (5) and (6), is a very simple property. But even if this claim is true, our intuitions are fallible, and therefore they do not confer certainty on (6).

The foregoing truths open the way for the further development of the third objection to the Plip-based argument. Let us treat There is a God as identical with $\mathrm{T}$, and let us ignore the first objection. Let us concede to Swinburne, for the sake of discussion, the three suppositions that (3) is true, that (1) entails (2), and that (2) and (3) jointly entail (4). Why should Swinburne's case for (1) persuade us that There is a God is h-simpler than we would otherwise have thought? Why should we not instead conclude, in the light of the three conceded suppositions, that whatever considerations we have had for assigning a lesser degree of h-simplicity to There is a God - notably, the application of our criteria of h-simplicity directly to $\mathrm{T}$ - should persuade us that the property Plip is less simple than (1) asserts it to be? In the absence of a convincing answer to this question, the Plip-based argument does not provide strong grounds for accepting (4). ${ }^{39}$

\footnotetext{
${ }^{39}$ I thank Richard Swinburne for helpful discussion of an earlier version of this paper.
} 